\title{
Rare cancers are common: time to act
}

Editorial

Michalis Liontos ${ }^{1 *}$, Evangelos Karamitrousis², Nikolaos Tsoukalas ${ }^{3}$, Ioannis Boukovinas ${ }^{4}$

${ }^{1}$ Department of Clinical Therapeutics, National and Kapodistrian

University of Athens, Alexandra Hospital, Athens, Greece

2Department of Medical Oncology, Aristotle University of Thessaloniki,

Papageorgiou General Hospital, Thessaloniki, Greece

${ }^{3}$ Department of Oncology, 401 General Military Hospital, Athens, Greece

${ }^{4}$ Department of Oncology, Bioclinic, Thessaloniki, Greece

Rare cancer is a term based on the frequency of the disease. More specifically, the Surveillance of Rare Cancers in Europe (RARECARE) project proposed that cancers should be designated as rare when their incidence is lower than 6 per 100,000 population per year [1]. Despite though, each rare cancer is infrequently encountered within the population, rare cancers in total, currently affect 4 million people in the European Union. Furthermore, 500,000 patients are diagnosed with rare cancers, annually, representing $20 \%$ of new cancer diagnoses [2]. This number far exceeds annual lung cancer diagnoses (approximately 320,000), indicating that a special interest should be given to this group of cancers.

Rare cancers are characterized by specific challenges, both for clinicians and the health systems in general. Patients with rare cancers have lower 5-year survival rates and less improvement in survival the last two decades, in comparison to those with more common cancers [3]. This reflects the lack of experience with rare cancers among clinicians, having as a consequence, not only delays in the diagnosis, but also uncertainty in the management of these patients. Usually, there are very few experts for rare cancers who could provide advice on diagnosis and management. In most of the countries studied, centers of expertise are rare, and these cases are diluted within the community. As a consequence, the interest for research in rare cancers is lacking, there are limited registries of rare cancer cases and corresponding biobanks that could support research efforts. The research in the field of rare cancer suffers the same fragmentation of areas in general health system, but the consequences are more pronounced here.
All of the above are translated in limited opportunities to perform clinical trials in rare cancers and also restrictions in funding.

Responding to the unmet need for improvement of care of rare cancer patients, European Union has launched Rare Cancer Europe, an initiative in association with scientific societies, patient's coalitions, European comprehensive cancer centers, and industry partners. The scope of this initiative is to promote establishment of centers of excellence across the continent and to tackle the barriers imposed in rare cancer care and the access of the patients to specialized treatments. To address these issues, several reference networks for rare diseases have been formed within the European Reference Network program, including European Rare Adult Solid Cancers network (EURACAN), PaedCan (pediatric cancers), and EuroBloodNet (hematological diseases and cancers). Now, we must go ahead addressing the major factors that can affect survival rates for rare cancers. These include the difficulties in carrying out clinical trials, due to the small number of patients with these cancers, as well as discrepancies in quality of care due to limited clinical expertise in the community.

Recently, the Priority Medicines (PRIME) scheme was launched by the EMA to enhance support for the development of medicines that target an unmet medical need. Through PRIME, the EMA offers early and proactive support to medicine developers to optimize the generation of robust data on a medicine's benefits and risks and enable accelerated assessment of medicine's applications.

Among stakeholders implicated in management of patients with rare cancers, education of health-care 
professionals and access to information from patients are considered as of primary importance to improve survival. The overall aim is to create both medical education and career pathways for young oncologists interested in managing rare cancers, particularly rare adult solid tumors.

In this issue of Forum of Clinical Oncology, we respond to the need for information regarding rare cancers and their proper management, with two interesting cases and corresponding reviews of the literature $[4,5]$. We believe that this could be the cornerstone for an open dialogue, regarding the proper organization of reference centers for rare cancers in Greece and their interconnection with European and global networks. Rare cancers are more common than most of us believe. It is time to act, and we should act now.

\section{References}

[1] Casali P.G. and A. Trama, Rationale of the rare cancer list: a consensus paper from the Joint Action on Rare Cancers (JARC) of the European Union (EU). ESMO open, 2020. 5(2): e000666.

[2] EU. [cited 2020 26/11/2020]; Available from: rarecacner.eu.

[3] Gatta G., et al., Burden and centralised treatment in Europe of rare tumours: results of RARECAREnet\&\#x2014; a population-based study. The Lancet Oncology, 2017. 18(8): 1022-1039.

[4] Pourtsidis A., et al. Acinar cell carcinoma in childhood: A case report of a very rare tumor. Forum Clin Oncol. 2020; 11(2):3-8.

[5] Mohammad A, et al. Targeted therapy in advanced desmoid tumors: Current Perspectives. Forum Clin Oncol. 2020; 11(2):9-16. 